\title{
Let's Not Forget About Regional Therapy for the Treatment of Locally Advanced Extremity Melanoma
}

\author{
Giorgos C. Karakousis, MD, FACS \\ Department of Surgery, Hospital of the University of Pennsylvania, Philadelphia, PA
}

In-transit metastases (ITM) are estimated to occur as recurrence in approximately $4-11 \%^{1,2}$ of patients with melanoma and represent a distinct pattern of disease spread characterized by dermal or subcutaneous metastases within lymphatics. They often pose a significant clinical challenge in their treatment, and can have a substantial impact on patient quality of life, being a source of bleeding, infection, discomfort, and impaired limb function. From the late 1950s, isolated limb perfusion (ILP) of high-dose chemotherapy to the involved extremity was developed as a limb-salvage method to address this clinical disease pattern. ${ }^{3}$ In the early 1990s, Thompson et al. ${ }^{4}$ in Australia developed isolated limb infusion (ILI) as a less morbid, shorter procedure length, and more cost-effective approach for percutaneous access delivery of chemotherapy to an extremity, obviating the need for a cardiopulmonary bypass circuit. These techniques, offered at specialized centers, were conceptually particularly appealing in a prior era of largely ineffective systemic therapies, as they could elicit appreciable in-field response rates while largely sparing patients from systemic toxicity.

Within the past decade, the field of melanoma has seen the almost dizzying discovery and approval of multiple new drug and drug combination therapies, including immune checkpoint inhibitors, targeted therapies, and local intralesional therapy (talimogene laherparepvec). Many of these treatments have naturally made their way to earlier stage III disease in the adjuvant setting and in the treatment of advanced stage III disease, including in the management of patients with surgically unresectable in-transit disease.

(C) Society of Surgical Oncology 2019

First Received: 14 April 2019;

Published Online: 6 May 2019

G. C. Karakousis, MD, FACS

e-mail: giorgos.karakousis@uphs.upenn.edu
This has left regional therapy (ILP, ILI) as just one of a multitude of approaches to be considered in the treatment of a specific patient with ITM. The advent of these welcomed therapeutics has brought alongside a multitude of new questions: What is the optimal initial approach to patients with ITM without distant metastases? For which patients with ITM is regional therapy ideally suited? Can regional and systemic therapies be combined to provide synergy for improved regional and systemic efficacy and how so?

In this issue of Annals of Surgical Oncology, Miura et al. ${ }^{5}$ present a large multicenter (including nine centers in the USA and Australia) retrospective experience of ILI in patients with stage IIIB/IIIC melanoma and extremity ITM. The study includes 687 patients with long-term follow-up undergoing first-time ILI with melphalan and actinomycin $\mathrm{D}$, reporting a complete response $(\mathrm{CR})$ rate of $28.9 \%$ with an overall response (OR) rate of $64.1 \%$. Factors significantly associated with both in-field progression-free survival (IPFS) and distant disease progression-free survival (DPFS) on multivariate analysis included stage of disease and response to treatment. With median follow-up of nearly 4 years, patients with CR to ILI demonstrated median IPFS of 36.7 months and DPFS of 101.7 months. By comparison, IPFS and DPFS were noted to be 13.5 and 26.3 months in patients with partial response; and in those with progressive disease after ILI, DPFS was only 10.1 months. CR remained significantly associated with improved overall survival, in addition to other tumor factors (stage IIIB status, lower burden of disease, and lower thickness primary tumor). Severe toxicity associated with ILI treatment was relatively low, with only $3.9 \%$ of patients experiencing Wieberdink toxicity score ${ }^{6}$ of IV (threatened or actual compartment syndrome) with no patients undergoing amputation. 
There are some notable limitations to the current study worth mentioning, which the authors largely acknowledge. Its retrospective design is subject to potential influences of patient heterogeneity and patient selection. Time to development of ITM after treatment of the primary melanoma or duration of ITM prior to consideration of ILI therapy, for instance, are but some factors of tumor biology which could influence the decision for offering regional therapy and impact disease-specific outcomes that are not captured in the current study. Moreover, since the study spans a long time period (1992-2018), there is significant variability in the systemic therapies patients may have received prior or subsequent to ILI treatment, which could influence survival outcomes; these are also not captured in the present study. Nonetheless, the authors importantly reaffirm in this large experience previously reported objective response rates to ILI therapy which are very compelling, particularly when taken in the context of response rates to some of the novel systemic therapies and their associated toxicities. Moreover, the strong association demonstrated between treatment response and not only regional control but also distant disease progression-free survival is intriguing, offering insight into which patients may potentially be spared systemic therapy after regional therapy.

As the authors note in their discussion, current National Comprehensive Cancer Network (NCCN) guidelines ${ }^{7}$ recognize multiple treatment options for ITM in melanoma, including systemic therapies (immune checkpoint inhibitors, targeted therapies), intralesional therapy, surgery when feasible, topical therapy (imiquimod) for surface dermally based lesions, radiation, and regional therapy (ILP, ILI). In the absence of prospective data directly comparing modalities for ITM disease, large multicenter studies such as the present are important in providing efficacy and morbidity data to help inform clinical decisions and counseling of these patients. Ultimately, a combination of different approaches may offer synergy in treatment outcomes surpassing any single therapy approach. In a phase II trial by Ariyan et al., ${ }^{8} 26$ patients with in-transit extremity melanoma and stage IIIB-IV disease were treated with melphalan ILI in combination with systemic CTLA4 blockade (ipilimumab). CR was noted to be an impressive $62 \%$ with OR of $85 \%$. While limb-specific toxicity did not appear to be increased in the trial with combined therapy, $38 \%$ of patients were noted to have a grade 3 or 4 treatmentrelated adverse event at the $10 \mathrm{mg} / \mathrm{kg}$ ipilimumab dose used, and only $54 \%$ of patients received all four planned induction doses of CTLA4 blockade.

The authors Miura et al. should be commended for reminding us through the current study of the efficacy and utility of regional therapy and ILI specifically in this evergrowing and evolving landscape of treatments for metastatic melanoma. It is important that consideration of regional therapy approaches continues to take place for appropriate patients in the context of multidisciplinary discussions and not be left as an afterthought - that is, after other options have failed. Rather, these modalities should be discussed and considered early in the disease course of ITM, when disease burden is lower and these approaches are more likely to be effective. Ultimately, as surgical oncologists, we must continue to try to better understand which patients are ideally suited for regional therapy and the optimal timing of such treatment. Development of novel biomarkers that can add to already studied patient and tumor prognostic factors will no doubt be important in this endeavor. Such biomarkers can help prognosticate response but also better characterize a "lymphotropic" disease pattern for which regional therapy may be particularly relevant. Moreover, further efforts to synergize regional and systemic approaches with acceptable toxicity can offer new opportunities to improve outcomes in patients with locally advanced disease. Recognizing regional therapy not only as a method of delivering directed cytotoxic therapy to areas where clinical disease is most apparent but also as a potentially important immunopriming modality which can potentiate other immunotherapy strategies when used in combination opens the door to further new drug discovery in regional treatment. For sure, much exciting work still lies ahead in understanding how to optimally sequence and integrate regional and systemic therapies in order to provide the best possible outcome for the individual patient with ITM.

\section{REFERENCES}

1. Read RL, Haydu L, Saw RP, et al. In-transit melanoma metastases: incidence, prognosis, and the role of lymphadenectomy. Ann Surg Oncol. 2015;22:475-581.

2. Umakanthan JM, Marr A. Management of in transit melanoma: present challenges and future directions. $J$ Oncol Pract. 2018;5:304-5.

3. Creech JO, Krementz ET, Ryan RF, et al. Chemotherapy of cancer: regional perfusion utilizing an extracorporeal circuit. Ann Surg. 1958;148:616-32.

4. Thompson JF, Waugh RC, Saw RPM, Kam PCA. Isolated limb infusion with melphalan for recurrent limb melanoma: a simple alternative to isolated limb perfusion. Reg Cancer Treat. 1994;7:188-92.

5. Miura JT, Kroon HM, Beasley GM, et al. Long-term oncologic outcomes after isolated limb infusion for locoregionally metastatic melanoma: an international multicenter analysis. Ann Surg Oncol. 2019. https://doi.org/10.1245/s10434-019-07288-w.

6. Wieberdink J, Benckhuysen C, Braat RP, van Slooten EA, Olthuis GA. Dosimetry in isolation perfusion of the limbs by assessment of perfused tissue volume and grading of toxic tissue reactions. Eur J Cancer Clin Oncol. 1982;18:905-10.

7. NCCN Clinical Practice Guidelines in Oncology: National Comprehensive Cancer Network. 2019. https://www.nccn.org/profe ssionals/physician_gls. 
8. Ariyan CE, Brady MS, Siegelbaum RH, et al. Robust antitumor responses result from local chemotherapy and CTLA4-blockade. Cancer Immunol Res. 2018;6:189-200.
Publisher's Note Springer Nature remains neutral with regard to jurisdictional claims in published maps and institutional affiliations. 